\section{Inflammasome 'prionization'}

The inflammasome is often a ternary complex composed of a sensor molecule, an adaptor and a caspase. Two papers in Cell, by Egelman et al. and Chen et al., throw light on how the AIM2 and NLRP3 inflammasomes assemble. Activation of either inflammasome results in nucleation of the adaptor ASC by components of the sensors AIM2 and NLRP3. Structural and electromicroscopical analysis demonstrates that the nucleated ASC then polymerizes into a structure with distinct prion-like properties. Assembled inflammasomes are known to cleave the pro-form of the inflammatory cytokine interleukin $1 \beta$ (IL-1 $\beta$ ), and these papers demonstrate by mutagenesis that the formation of prion-like ASC fibers is critical for this activity. 'Prionization' may therefore be an essential and unifying step in the generation of many physiological inflammatory responses. Cell 156, 1193-1206 \& 1207-1222 (2014)

\section{Human skin $\mathrm{T}_{\text {reg }}$ cells}

Regulatory $\mathrm{T}$ cells ( $\mathrm{T}_{\text {reg }}$ cells) are present in tissues throughout the body, where they serve important roles in maintaining immunological homeostasis; however, few studies have examined human tissueresident $\mathrm{T}_{\text {reg }}$ cells. In the Journal of Clinical Investigation, Rosenblum et al. characterize a population of $\mathrm{T}_{\text {reg }}$ cells in human skin that accumulate progressively with age. Skin $\mathrm{T}_{\text {reg }}$ cells have a distinct effector-memory phenotype suggestive of previous antigen recognition. These $\mathrm{T}_{\text {reg }}$ cells are poorly migratory and thus seem to be a true skin-resident population 'preferentially' associated with hair follicles. Sequencing of T cell antigen receptors demonstrates that this population has a repertoire distinct from that of their conventional effector T cell counterparts. Finally, skin $\mathrm{T}_{\text {reg }}$ cells isolated from psoriatic lesions are more proliferative and express the inflammatory cytokine IL-17. Human skin therefore has a resident $\mathrm{T}_{\text {reg }}$ cell population that may be functionally defective under conditions of inflammation.

J. Clin. Invest. 124, 1027-1036 (2014)

\section{DC division of labor}

The contributions of various dendritic cell (DC) subsets to the generation of effector versus memory $C D 8^{+} \mathrm{T}$ cells remains unclear. In Immunity, Lin et al. show that lung $\mathrm{CD}_{103^{+}}$or CD11 b+ migratory DCs activate naive CD8 ${ }^{+} \mathrm{T}$ cells differently during infection with influenza virus. Through the use of mice deficient in $\mathrm{CD} 103^{+} \mathrm{DCs}$, the authors show this DC subset is required for the efficient generation of virus-specific CD8 ${ }^{+}$ $T$ cells with an effector or effector-memory phenotype in the lymph nodes and the migration of those cells to the lungs. However, the generation of $\mathrm{CD}^{+} \mathrm{T}$ cells with a central memory phenotype is supported mostly by the CD11b+ DC subset. CD103+ DCs have tenfold higher expression of CD24, which serves as a costimulatory ligand for the differentiation of effector $\mathrm{CD}^{+}$ T cells at least in part through capture of the alarmin HMGB1 and engagement of the receptor RAGE on $\mathrm{CD}^{+} \mathrm{T}$ cells. Thus, different intrinsic properties of DCs dictate the outcome of the effector or memory differentiation of $\mathrm{CD}^{+} \mathrm{T}$ cells.

Immunity 40, 400-413 (2014)

Written by Laurie A. Dempsey, Zoltan Fehervari \& Ioana Visan

\section{Transcription concepts}

In response to inflammatory stimuli, the transcription factor NF- $\kappa \mathrm{B}$ promotes gene transcription. In Molecular Cell, Gaudet and colleagues use single-cell analysis to show that the transcription of NF- $\mathrm{KB}$-dependent genes is determined by the change in abundance, not the absolute abundance, of the NF- $\kappa \mathrm{B}$ subunit RelA in the nucleus. For tumor-necrosis factor-sensitive genes that are constitutively expressed and 'scaled up' or strictly inducible by NF- $\mathrm{KB}$, the maximum change in the abundance of nuclear RelA is the strongest predictor of transcription. A computational model that includes a 'competitor' that is subject to self-repression and has variable DNA-binding affinities for target promoters relative to those of the transcriptionally competent $\mathrm{NF}-\kappa \mathrm{B}$ reproduces the transcription patterns observed experimentally: with high competitor affinity, the transcription of target genes is inducible, whereas with low competitor affinity, gene expression is constitutive. The NF- $\kappa B$ subunits p50 and p52, which form transcriptionally incompetent homodimers, and the $\mathrm{NF}-\kappa \mathrm{B}$ inhibitor Bcl-3 fit the profile for such competitors. Thus, variation in the relative expression of competitors can vary the expression of 'hard-wired' promoters in various cell types. Mol. Cell 53, 867-879 (2014)

\section{Gut crosstalk}

The gut normally maintains immunological tolerance despite the enormous microbial load in the lumen. In Science, Merad and colleagues identify crosstalk between interdependent cells of the innate immune system that acts to maintain immunological quiescence in the gut. Tissue-resident macrophages sense gut microbes and secrete IL-1 $\beta$, which in turn induces ROR $\mathrm{t}^{+}$innate lymphoid cells to produce GM-CSF, a cytokine that supports the proliferation and function of macrophages. Mice that lack GMCSF have fewer colon-resident macrophages and DCs, produce less retinoic acid and less of the immunosuppressive cytokines IL-10 and TGF- $\beta$, which results in fewer $T_{\text {reg }}$ cells and impaired oral tolerance of dietary antigens. Notably, this tolerogenic crosstalk seems to be specific to the colon, as excess GM-CSF has pathogenic effects in other tissues. This finding thus warrants further elucidation of the environmental cues that underlie cellular crosstalk.

$L A D$

Science (13 March 2014) doi:10.1126/science.1249288

\section{Facilitating Nod detection}

Phagocytosed bacteria can activate host innate sensors that include membrane-tethered Toll-like receptors and cytosolic Nod-like receptors. A dilemma of membrane topology, however, raises questions about how Nod ligands can activate their respective receptors. In Nature, Mellman and colleagues identify the peptide transporters SLC15A3 and SLC15A4 as endolysosomal constituents that transport bacterial muramyl dipeptide across membranes to activate Nod2. DCs constitutively express SLC15A4, but they inducibly express SLC15A3 in response to lipopolysaccharide. Both proteins use proton gradients to drive the transport of peptides into the cytosol. Nod2 can associate with both transporters in the presence of muramyl dipeptide and trigger activation of the kinase RIPK2 and antimicrobial pathways. Thus, the SLC15A endolysosomal transporters facilitate the recognition of phagocytosed bacterial components. $L A D$ Nature (30 March 2014) doi:10.1038/nature13133 